\title{
Isolation of three novel reassortant phleboviruses, Ponticelli I, II, III, and of Toscana virus from field-collected sand flies in Italy
}

Mattia Calzolari ${ }^{1 *}$, Chiara Chiapponi ${ }^{1}$, Romeo Bellini ${ }^{2}$, Paolo Bonilauri ${ }^{1}$, Davide Lelli ${ }^{1}$, Ana Moreno ${ }^{1}$, Ilaria Barbieri ${ }^{1}$, Stefano Pongolini ${ }^{1}$, Antonio Lavazza ${ }^{1}$ and Michele Dottori ${ }^{1}$

\begin{abstract}
Background: Different phleboviruses are important pathogens for humans; most of these viruses are transmitted by sand flies. An increasing number of new phleboviruses have been reported over the past decade, especially in Mediterranean countries, mainly via their detection in sand flies.

Results: At least five different phleboviruses co-circulated in sand flies that were collected in three sites in EmiliaRomagna (Italy) in the summer of 2013. The well-known Toscana virus (TOSV) was isolated; three new, closely related phleboviruses differing in their $\mathrm{M}$ segments and tentatively named Ponticelli I, Ponticelli II and Ponticelli III virus, respectively, were isolated; a fifth putative phlebovirus, related to the sand fly fever Naples phlebovirus species, was also detected. The co-circulation, in a restricted area, of three viruses characterized by different $M$ segments, likely resulted from reassortment events. According to the phylogenetic analysis of complete genome sequences, the TOSV belongs to clade A, together with other Italian isolates, while the Ponticelli viruses fall within the Salehabad phlebovirus species.

Conclusions: Results highlight an unexpected diversity of phleboviruses that co-circulate in the same area, suggesting that interactions likely occur amongst them, that can present challenges for their correct identification. The co-circulation of different phleboviruses appears to be common, and the bionomics of sand fly populations seem to play a relevant role. Such a complex situation emphasizes the need for detailed investigations of the biology of these viruses to better characterize their pathogenic potential for mammals, including humans.
\end{abstract}

Keywords: Phlebovirus, Ponticelli I virus, Ponticelli II virus, Ponticelli III virus, Toscana virus, Fermo-like virus, Sand fly, Phlebotomus perfiliewi, Phlebotomus perniciosus

\section{Background}

Sand flies are hematophagous dipterans with crepuscular/nocturnal activity and a resting behaviour during daytime. Their biology is largely enigmatic due to focal distribution in occupied areas linked to the availability of breeding sites and hosts and fluctuating abundance during the active season; both characteristics hamper their sampling $[1,2]$.

\footnotetext{
* Correspondence: mattia.calzolari@izsler.it

${ }^{1}$ Istituto Zooprofilattico Sperimentale della Lombardia e dell'Emilia-Romagna

"Bruno Ubertini", Brescia, Italy

Full list of author information is available at the end of the article
}

Although sand flies are fragile, small flyers that are unable to disperse over long distances, they are important to human and animal health due to their capacity to transmit a wide range of pathogens, including protozoa such as Leishmania parasites and different viruses belonging to the genera Phlebovirus, Vesiculovirus and Orbivirus [2].

Among sand fly-borne viruses, the genus Phlebovirus of the family Phenuiviridae (previously included in the family Bunyaviridae), is the most important group affecting human and animal health. Most of the known phleboviruses are transmitted by sand flies, while others 
are transmitted by mosquitoes and ticks (i.e. Rift Valley fever virus and Uukuniemi virus). Several sand fly phleboviruses are recognized as causes of disease in humans, such as sand fly fever or "three-day fever", the selflimiting influenza-like syndrome caused by the Sicilian and Naples viruses, and summer meningitis, often caused by the Toscana virus (TOSV) [3].

Phleboviruses are characterized by a tri-segmented negative-stranded RNA genome including an L segment (encoding the RNA-dependent RNA polymerase), an M segment (encoding the two glycoproteins, Gn and Gc) and an ambisense $S$ segment (encoding the nucleocapsid protein, N, and a smaller non-structural protein, NS) [4].

According to the International Committee on Taxonomy of Viruses [5], the genus Phlebovirus includes ten viral species in the Old World and the Americas. These species are defined by serological relationships in neutralization tests [6], but the discovery of several new phleboviruses brought attention to the need to introduce new criteria for phlebovirus classification [7]. Moreover, the classification of these viruses is complicated by their reassorting ability, particularly for the $M$ segment, as already reported between related sand fly phleboviruses in South America [7] and, more rarely, in the Old World $[8,9]$.

Over the past decade, a wide variety of previously unreported phleboviruses were detected, particularly in the Mediterranean basin, including Sicilian-like viruses, e.g. the sand fly fever Cyprus virus [10], the sand fly fever Turkey virus [11], a phlebovirus from Algeria [12], and the Toros virus in Turkey [13]. Also, sequences of putative viruses related to this group were detected in Cyprus and Tunisia and tentatively named Girne 2 virus [14] and Utique virus [15], respectively. Different phleboviruses of the sand fly fever Naples phlebovirus species were also isolated, such as the Massilia and Granada viruses in France and Spain, respectively $[8,16]$, the Punique virus in Tunisia [15], the Fermo virus in Italy [17], the Arrabida virus in Portugal [9] and the Zerdali virus in Turkey [13]. Furthermore, some putative viruses were detected in France, Cyprus and Tunisia, and tentatively named Provencia [18], Girne 1 virus [14] and Saddaguia virus [19], respectively. The phleboviruses Adana [20], Alcube [21] and Medjerda Valley [22], attributable to the Salehabad phlebovirus species, were isolated and characterized in Turkey, Portugal, and Tunisia, respectively. Sequences related to putative viruses of the latter group were also detected in field-collected sand flies in Albania and Turkey and named Adria virus [23] and Edirne virus [14], respectively.

This study aimed to describe the phlebovirus diversity in sand flies sampled in 2013 in three sites within the hilly area of the Emilia-Romagna region (northern Italy) where TOSV was previously detected in 2012 [24].

\section{Methods}

\section{Sampled sites}

Three sites in which TOSV was detected in 2012 [24] were sampled in 2013. The coordinates of the sampled sites are as follows: site 1: $44^{\circ} 9^{\prime} 56^{\prime \prime} \mathrm{N}, 11^{\circ} 58^{\prime} 9^{\prime \prime} \mathrm{E}$; site 2: $44^{\circ} 19^{\prime} 18^{\prime \prime} \mathrm{N}, 1^{\circ} 38^{\prime} 28^{\prime \prime} \mathrm{E}$; site 3: $44^{\circ} 17^{\prime} 15^{\prime \prime} \mathrm{N}, 11^{\circ} 41^{\prime} 46^{\prime \prime} \mathrm{E}$ (Fig. 1). Site 1 was sampled twice, whereas the other sites were sampled only once. As previously described [24], sampling stations were located in the hilly area of the Emilia-Romagna region (altitude between 128 and 207 masl.). This area was characterized by cultivated fields bordered by hedges, woodlands, and badlands (Additional file 1: Figure S1).

\section{Sand fly sampling and identification}

To collect the largest number of specimens, samples were taken in summertime, the more favourable season for sand flies in the surveyed region. Sand fly sampling was performed using carbon-dioxide-baited traps operating overnight. To maximize the number of collected sand flies, 2 to 5 traps with a minimum distance of $10 \mathrm{~m}$ were utilized for each site (Table 1). A subset of captured sand flies was clarified in chlorolactophenol and morphologically identified under a light microscope according to specific morphological keys $[25,26]$. The remaining sand flies were stored in dry ice and used within a maximum of 5 days for virus isolation in cell culture.

\section{Virus isolation and purification on cell cultures}

Collected sand flies were pooled by collection site; to minimize handling time (thus avoiding temperature fluctuations), and maximize the chances of isolation, large pools containing both males and females were assembled. Pools were homogenized in minimal essential medium, supplemented with penicillin and streptomycin, using a sterilized glass potter and clarified by centrifugation at $3000 \times g$ for $15 \mathrm{~min}$. Samples were inoculated in a confluent monolayer of VERO cells (African green monkey kidney cells) at passage 170 (cell culture biobank of IZSLER, code BSCL86), incubated at $37{ }^{\circ} \mathrm{C}$ with $5 \% \mathrm{CO}_{2}$ and observed daily for 7 days to observe the development of cytopathic effects (CPE). In the absence of CPE, the cryolysates were subcultured twice into fresh monolayers.

Isolated viruses were cultured in VERO cells under previously reported conditions and observed daily for 7 days, to characterize CPE. To exclude the presence of 2 or more viral strains in culture, selected isolates were cloned by plaque purification by 2 rounds of serial dilutions in 96-well plates (maximum dilution of $10^{-8}$ ). One of the obtained clones was re-cultured on VERO cells and subjected to sequencing. 


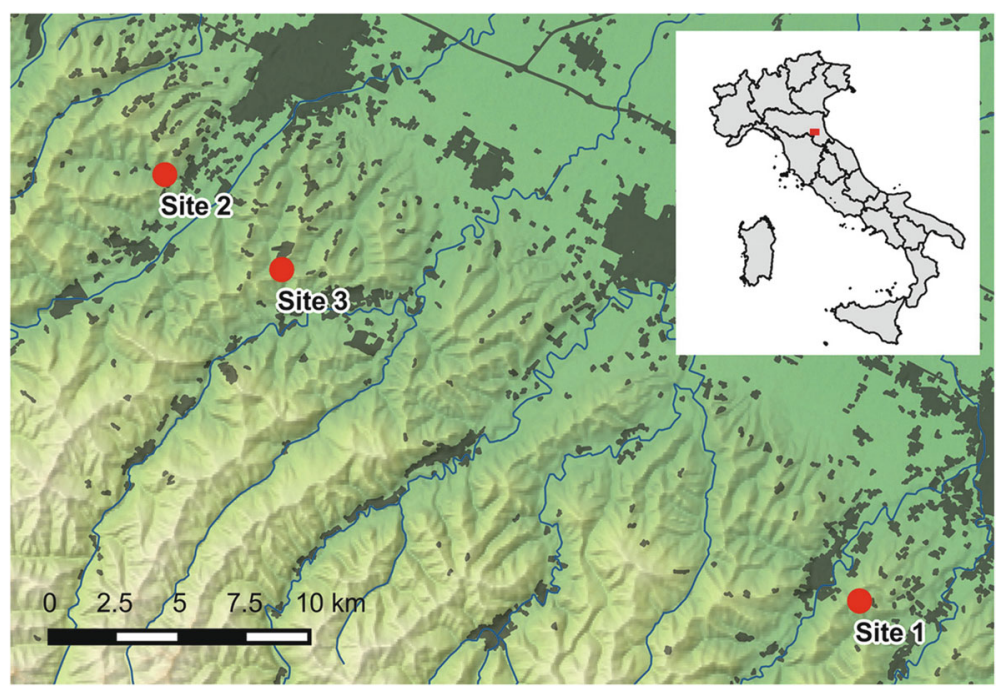

Fig. 1 Physical map with locations of surveyed sites and position of surveyed area (red square) on a map of Italy. Urbanized areas are in gray

\section{Virus detection and sequencing}

Viral RNA was extracted from supernatant cultures that displayed CPE using TRIzol LS Reagent (Invitrogen, Carlsbad, CA, United States); cDNA synthesis was performed using random hexamers (Roche Diagnostics, Mannheim, Germany) and the SuperScriptH II reverse transcriptase (Invitrogen, Carlsbad, CA) and then tested by real-time RT-PCR for TOSV detection [27]. Samples negative for TOSV were subjected to PCR for the detection of phleboviruses (Pan-Phlebo-PCR), usibg by one reverse and two forward primers and targeting a 370nucleotide region of the $S$ segment [28]. The obtained amplicons were sequenced, and the sequences were utilized for virus identification by BLAST analysis against the GenBank database (https://blast.ncbi.nlm.nih.gov/ Blast.cgi).

The selected viruses isolated from the cell culture supernatant were sequenced on a MiSeq Instrument (Illumina Inc., San Diego, CA, USA). To prepare DNA libraries for whole-genome sequencing, viral RNA was amplified by One-Step RT-PCR with the simultaneous amplification of all 3 viral segments. In-house-designed primers recognizing $5^{\prime}$ and $3^{\prime}$ regions of the 3 segments
L, $M$ and S, TOSV_uni (5'-GCC GGA GCT CTG CAG ATA TC, A CAC ArA GA-3') for TOSV and PhleboV_uni (5' -GCC GGA GCT CTG CAG ATA TC, A CAC ArA G-3') for phleboviruses, were used. To stabilize the primer in a subsequent amplification reaction by OneStep RT-PCR, a known oligonucleotide sequence was inserted in the $5^{\prime}$ region of the primer [29]. Briefly, $10 \mu \mathrm{l}$ of RNA was incubated with 200 pmol primers at $65{ }^{\circ} \mathrm{C}$ for $5 \mathrm{~min}$. On ice, $25 \mu \mathrm{l}$ of $2 \times$ master mix and $1 \mu \mathrm{l}$ of SuperScript III One-Step RT-PCR System with Platinum Taq DNA Polymerase were added to a final reaction volume of $50 \mu \mathrm{l}$. One-Step RT-PCR was performed with 1 cycle at $42{ }^{\circ} \mathrm{C}$ for $60 \mathrm{~min}$ and 1 cycle at $94{ }^{\circ} \mathrm{C}$ for 2 min. The PCR conditions were as follows: 5 cycles of denaturation at $94{ }^{\circ} \mathrm{C}$ for $30 \mathrm{~s}$, annealing at $45{ }^{\circ} \mathrm{C}$ for $30 \mathrm{~s}$, and extension at $68{ }^{\circ} \mathrm{C}$ for $6 \mathrm{~min}$, followed by $30 \mathrm{cy}$ cles with the above conditions and an annealing temperature of $60{ }^{\circ} \mathrm{C}$. PCR products were purified using NucleoSpin Gel and PCR Clean-up (Macherey-Nagel GmbH \& Co, Düren, Germany). DNA libraries were prepared using a NEXTERA-XT Kit (Illumina Inc., San Diego, CA, USA) according to the manufacturer's instructions. Pooled libraries were sequenced on a MiSeq

Table 1 Sand flies that were collected and tested during the summer in 2013 concerning identified species

\begin{tabular}{lllllll}
\hline Site & Date & Traps & Collected & P. perfiliewi (M/F) & P. perniciosus (M/F) & Tested/Pools \\
\hline Site 1 & $05-07-13$ & 5 & 14,974 & $482(40 / 442)$ & $13(0 / 13)$ & $14,479 / 25$ \\
& $23-08-13$ & 4 & 5682 & $452(47 / 405)$ & $8(0 / 8)$ & $5222 / 8$ \\
Site 2 & $16-07-13$ & 5 & 394 & $45(5 / 40)$ & $4(0 / 4)$ & $345 / 4$ \\
Site 3 & $23-07-13$ & 2 & 2079 & - & - & $2079 / 4$ \\
Total & & & 23,129 & $979(92 / 887)$ & $25(0 / 25)$ & $22,125 / 41$ \\
\hline
\end{tabular}


platform (Illumina Inc., San Diego, CA, USA) using a MiSeq Reagent Kit v2 in a 250-cycle paired-end run. Data were assembled de novo by the NextGen DNASTAR (DNASTAR, Madison, WI, USA) application and were analyzed using Lasergene Package software (v12.0). All obtained sequences were deposited in the GenBank database under the accession numbers KU573064KU573069 and KX388208-KX388225.

\section{Electron microscopy}

The supernatant fluids from cell cultures showing CPEs were subjected to negative staining electron microscopy using the Airfuge method [30]. Supernatants were ultracentrifuged (Airfuge, Beckman Coulter Inc. Life Sciences, Indianapolis, Indiana, USA) for $15 \mathrm{~min}$ at $82,000 \times g$ using a rotor holding six $175-\mu l$ test tubes in which specific adapters for $3 \mathrm{~mm}$ carbon-coated Formvar copper grids were placed. The grids were then stained using $2 \%$ sodium phosphotungstate $(\mathrm{pH}$ 6.8) for 1.5 min and observed under a Tecnai G2 Spirit Biotwin transmission electron microscope (FEI, Hillsboro, Oregon, USA) at $20,500-43,000 \times$ for at least $15 \mathrm{~min}$ before being considered negative. Attempts to identify the observed viral particles were based on their morphological characteristics.

\section{Phylogenetic analysis}

Obtained sequences were aligned with homologous sequences of other old-world phleboviruses transmitted by sand flies; these homologous sequences were retrieved from the GenBank database. Translated amino acid sequences were aligned using the ClustalW algorithm and alignments were refined manually; nucleotide sequences were utilized for phylogenetic analysis. The maximum likelihood approach was used to obtain a phylogenetic tree with the short-conserved sequence targets of the Pan-Phlebo PCR; the Kimura 2-parameter model with 5 discrete Gamma categories (model with the lowest Bayes factor) was selected with 1000 replicates of bootstrapping. Due to the wide diversity between complete sequences of phleboviruses, the neighbour-joining method (with distances computed using the LogDet method) was used to infer the phylogenetic tree with the $\mathrm{M}, \mathrm{L}$, and $\mathrm{S}$ sequences. Phylogenetic analysis, identity percentage among sequences and pairwise distance matrices were inferred using the MEGA 6 software [31]. Possible recombination events in Ponticelli sequences were checked with the Recombination Detection Program [32]. The coding sequences of the different genes (the RNA polymerase, the two glycoproteins, and the $\mathrm{N}$ and NS proteins) were manually concatenated, and the obtained chimeric genomes were screened by SIMPLOT software [33].

\section{Results}

Isolation and identification of viruses

A total of 23,129 sand flies were sampled in the 3 monitored sites, and a subsample of 1004 specimens was identified using morphological characteristics; of these, 979 were Phlebotomus perfiliewi and 25 were Phlebotomus perniciosus (Table 1). The remaining sand flies were pooled, depending on abundance, in 41 pools, and 23 of these pools showed a CPE on VERO cells (Table 2).

Cell culture supernatants with CPE were subjected to biomolecular analysis, and 4 of these samples, obtained from sand flies caught in site 1 on 2 distinct days, were TOSV positive using real-time RT-PCR; these samples were then directly subjected to complete genome sequencing (Table 2).

The presence of viruses causing CPE in TOSVnegative cell cultures were proved by electron microscopy which detected nearly spherical particles of 80 $100 \mathrm{~nm}$ with envelopes and small projections on the surface, morphologically resembling viruses of the Phenuiviridae family (Fig. 2). The same cell cultures (CPE present/TOSV-negative) were tested with Pan-Phlebo PCR, and all results were positive. Sequencing of the obtained amplicons yielded 24 sequences (Table 2), grouped in 2 separate clades in the phylogenetic tree (Fig. 3). Seventeen similar sequences were attributable to a new virus, tentatively named Ponticelli virus, according to the name of a village close to one of the sampling sites in which the virus was detected (GenBank: KY354371-KY354387). The alignment of 347 positions of 15 of these sequences showed an average identity of $99.0 \%$ (ranging between $97.7-100 \%$ ). These sequences clustered within a well-supported clade containing sequences of the Salehabad phlebovirus species in the phylogenetic tree (Fig. 3).

The remaining 7 sequences (alignable over $336 \mathrm{nu}$ cleotides, nt) were attributable to a second virus and showed an average identity of $95.1 \%$ (ranging from $100 \%$ to $89.9 \%$ ) (GenBank: KY354388-KY354394). These sequences clustered within a well-supported clade including the sand fly fever Naples phlebovirus species. Interestingly, 132 nucleotides of this last group of sequences partially overlapped with 3 sequences of the Fermo virus (GenBank: LT575235LT575237). In fact, these 2 groups of sequences showed an average identity of $97.0 \%$, with values ranging between $96.2-97.7 \%$.

\section{Full genome sequences}

Deep sequencing was applied to the 4 isolates with TOSV-positive PCR results. For 2 of these isolates, a TOSV genome was obtained, while for the remaining 2 , a genome attributable to the Ponticelli virus was obtained. Identity from $99.8 \%$ to $100 \%$ was obtained 
Table 2 Virological results of pools showing CPE, with reference to GenBank accession numbers of obtained sequences

\begin{tabular}{|c|c|c|c|c|c|}
\hline Sampling & Pools & TOSV PCR & Phlebo PCR & Ponticelli virus & Fermo-like virus \\
\hline \multirow[t]{14}{*}{ Site $1 / \mathrm{Jul}$} & $181135-1$ & - & + & KY354371 & KY354388 \\
\hline & $181135-2$ & - & + & KY354372 & KY354389 \\
\hline & 181135-3 & - & + & KY354373 & \\
\hline & $181135-4$ & - & + & & KY354390 \\
\hline & $181135-8$ & - & + & KY354374 & \\
\hline & $181135-9$ & - & + & KY354375 & KY354391 \\
\hline & $181135-10$ & - & + & KY354376 & \\
\hline & $181135-12$ & - & + & & KY354392 \\
\hline & $181135-13$ & - & + & KY354377 & \\
\hline & $181135-14$ & + & $\mathrm{np}$ & & \\
\hline & $181135-15$ & + & $\mathrm{np}$ & & \\
\hline & $181135-16$ & - & + & KY354378 & KY354393 \\
\hline & $181135-17$ & - & + & KY354379 & KY354394 \\
\hline & $181135-22$ & - & + & KY354380 & \\
\hline \multirow[t]{6}{*}{ Site 1/Aug } & 220116-1 & - & + & KY354381 & \\
\hline & $220116-2$ & - & + & KY354382 & \\
\hline & $220116-3$ & + & $\mathrm{np}$ & & \\
\hline & $220116-5$ & + & $\mathrm{np}$ & & \\
\hline & $220116-6$ & - & - & KY354383 & \\
\hline & $220116-8$ & - & + & KY354384 & \\
\hline Site $2 / J u l$ & $189826-4$ & - & + & KY354385 & \\
\hline \multirow[t]{2}{*}{ Site 3/Jul } & $194246-2$ & - & + & KY354386 & \\
\hline & $194246-4$ & - & + & KY354387 & \\
\hline
\end{tabular}

Abbreviation: $n p$ not performed

between sequences of the different segments of the 2 TOSV isolates: more precisely, $100 \%$ for $3413 \mathrm{nt}$ of the $M$ segment (GenBank: KU573065, KU573068), 99.8\% for 5953 nt of the L segment (GenBank: KU573064, KU573067) and 99.8\% for 1632 nt of the S segment (GenBank: KU573066, KU573069). In the phylogenetic tree obtained, sequences of all segments fell within the already described lineage $\mathrm{A}$, along with other Italian sequences [13, 14].

Other 4 isolates from site 1 and site 3 were selected and subjected to deep sequencing, which resulted in Ponticelli virus genome identification and increased the number of Ponticelli virus sequences obtained to 6. All the obtained sequences of the $\mathrm{L}$ segment (GenBank:
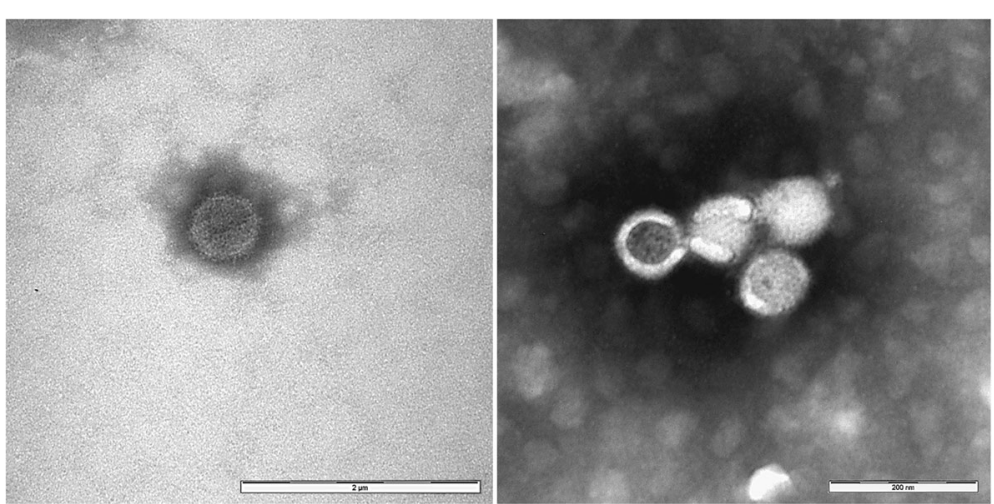

Fig. 2 Ultramicrograph of virions observed in the supernatant of VERO cells inoculated with ground sand flies. Round particles of 80-100 nm with envelopes and tiny projections on the surface are shown. Negative staining of NaPt 2\% (pH 6.8) observed with a TEM FEl Tecnai G2 Spirit Bio-twin 
98 EF120631 TOSV GR40 Spain FJ153285 TOSV ESH Spain KC776214 TOSV MRS2010-4319501 France KU204981 TOSV Nice/113 France KX010932 TOSV 54441132704 France KU204975 TOSV P233 France KU204978 TOSV TOSVIP51 France EF656361 TOSV AR France

99 FJ153286 TOSV H/IMTSSA France KM275763 TOSV/Portugal EF201833 TOSV ELB Portugal KU925898 TOSV 11368 France KP694242 TOSV A189 Algeria

97 KU935738 TOSV 5904242804 France

KM275772 TOSV/Pesaro.ITA/1992 Italy KM275237 TOSV/Siena.ITA/1984 Italy NC006318 TOSV Italy

JX867536 TOSVT152 Tunisia KM275768 TOSV/Macerata.ITA/1990/1 Italy JF330275 TOSV SI-121487 Italy KM275776 TOSV/Siena.ITA/1994 Italy AJ811547 Sabin Oct- 85 Italy KM275782 TOSV/Firenze.ITA/2009/5 Italy FJ848987 TOSV SI-121283 Italy KM275785 TOSV/Firenze.ITA/1981 Italy HM566178 SFNV-like virus Poona

77 EF201829 SFNV Sabin Italy

77 - EF201828 SFNV NAMRU 840055 Egypt

88 HM566182 SFVN

98 EF201832 SFNV R-3 Cyprus

EF201831 YU8-76 former Yugoslavia

JF939848 Tehran virus I-47 Iran

KP966618 Zerdali virus 37 Turkey

82 86 KY354392 181135-12

KY354390 181135-4

KY354394 181135-17

94 KY354391 181135-9

KY354391 181135-9

KY354388 181135-1
KY354393 181135-16

KY354389 181135-2

8648987 Punique virus PIB4 Tunisia

KP863801 Arrabida virus PoSFPhlebV/126/2008

KT906103 Massilia virus 130 France

89 KT906102 Massilia virus 127 France

GU135608 Granada virus GR25 Spain

84. GQ847511 SFSV Izmir19 Turkey

EU725773 Massilia virus W France

83. KM042104 SFSV Ethiopia-2011

EF201825 SFSV RM-09 Cyprus

EF201826 SFSV R-18 Cyprus

92 GU119908 SFSV Cyprus

EF201822 SFSV Sabin Italy

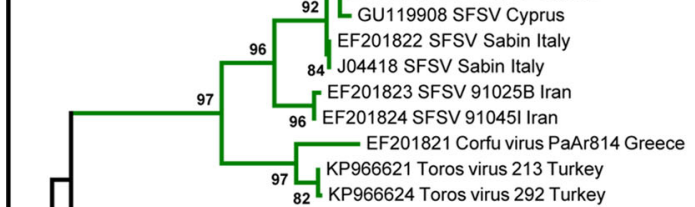

KF297914 Karimabad virus I-58 Iran KF297911 Karimabad virus AG Iran

99 KF297908 Karimabad virus P Iran

99 JX472402 Arbia ISS PHL18 Italy

Prus T131 Tunisia

86 KR363192 Alcube virus S20 Portugal

- JX472405 Salehabad virus I81 Iran

99

KJ939332 Adana virus Turkey

KY354371 181135-1 2

KY354384 220116-8

72 KY354374 181135-8

KY354375 181135-9 2

KY354380 181135-22

KY354381 220116-1

KY354377 181135-13

KY354379 181135-17 2

KY354382 220116-2

KY354387 194246-4

72 KY354373 181135-3

KY354385 189826-4

KY354386 194246-2

KY354376 181135-10

0.5

KY354383 220116-6

Fig. 3 (See legend on next page.) 
(See figure on previous page.)

Fig. 3 Phylogenetic tree of the field-detected S-segment sequences obtained by Pan-Phlebo PCR [28] and homologous sequences of Old-World sand fly-transmitted phleboviruses, obtained with the maximum likelihood method. Red: Salehabad phlebovirus species; green: sand fly fever Sicilian phlebovirus species; brown: Karimanbad virus; yellow, sand fly fever Naples phlebovirus species; sequences from this study in bold. Only bootstrap values over 70\% are shown near the respective branches. Abbreviations: SFNV, sand fly fever Naples virus; SFSV, sand fly fever Sicilian virus; TOSV, Toscana virus

KX388211, KX388214, KX388217, KX388223, KX3882 20 and KX388208) and of the S segment (GenBank: KX388213, KX388216, KX388219, KX388225, KX388 and KX388210) were closely related: the average identity of 5442 nucleotides of the $\mathrm{L}$ segment sequences was 98.7\% (ranging from $98.3 \%$ to $99.6 \%$ ); the average identity of 1266 aligned nt of the $\mathrm{S}$ segment sequences was 99.7\% (ranging from $98.0 \%$ to $99.3 \%$ ). Among the $\mathrm{M}$ segment sequences, 4 showed high rates of identity, i.e. the alignment of $4063 \mathrm{nt}$ revealed an average identity of 98.8\%, ranging from $98.5 \%$ to $99.4 \%$ (GenBank: KX388212, KX388215, KX388218 and KX388224). In contrast, the other 2 M segments (GenBank: KX388209 and KX388221, 583 nt sequence) differed from each other (identity 73.9\%) and the other isolates (average identity of $75.8 \%$ and $76.5 \%$, respectively). Therefore, $3 \mathrm{M}$ segments were observed and differentiated into 3 viruses tentatively named
Ponticelli I, Ponticelli II and Ponticelli III virus, respectively.

The phylogenetic analysis of the 3 segments showed that the Ponticelli viruses clustered together with sequences of viruses of the Salehabad phlebovirus species. The $\mathrm{L}$ and $\mathrm{S}$ segment trees shared a similar topology, in which Ponticelli viruses were more similar to the Adana virus (Fig. 4a, b, Additional file 2: Figure S2a). In the $M$ segment trees obtained using the complete alignment, the one based on the shortest $M$ segment available or sequences encoding the 2 glycoproteins ( $\mathrm{Gn}$ and $\mathrm{Gc}$ ), the viruses were closer to the Salehabad and Medjerda Valley viruses (Fig. 4c, Additional file 2: Figure S2b-d). The pairwise distances obtained for the $\mathrm{L}$ and $\mathrm{S}$ segments confirmed the Adana virus as the closer virus to the Ponticelli viruses. The nucleotide sequences differed by $19.6 \%$ in the $\mathrm{L}$ segment, $16.5 \%$ in the $\mathrm{N}$ gene, and $17 \%$ in

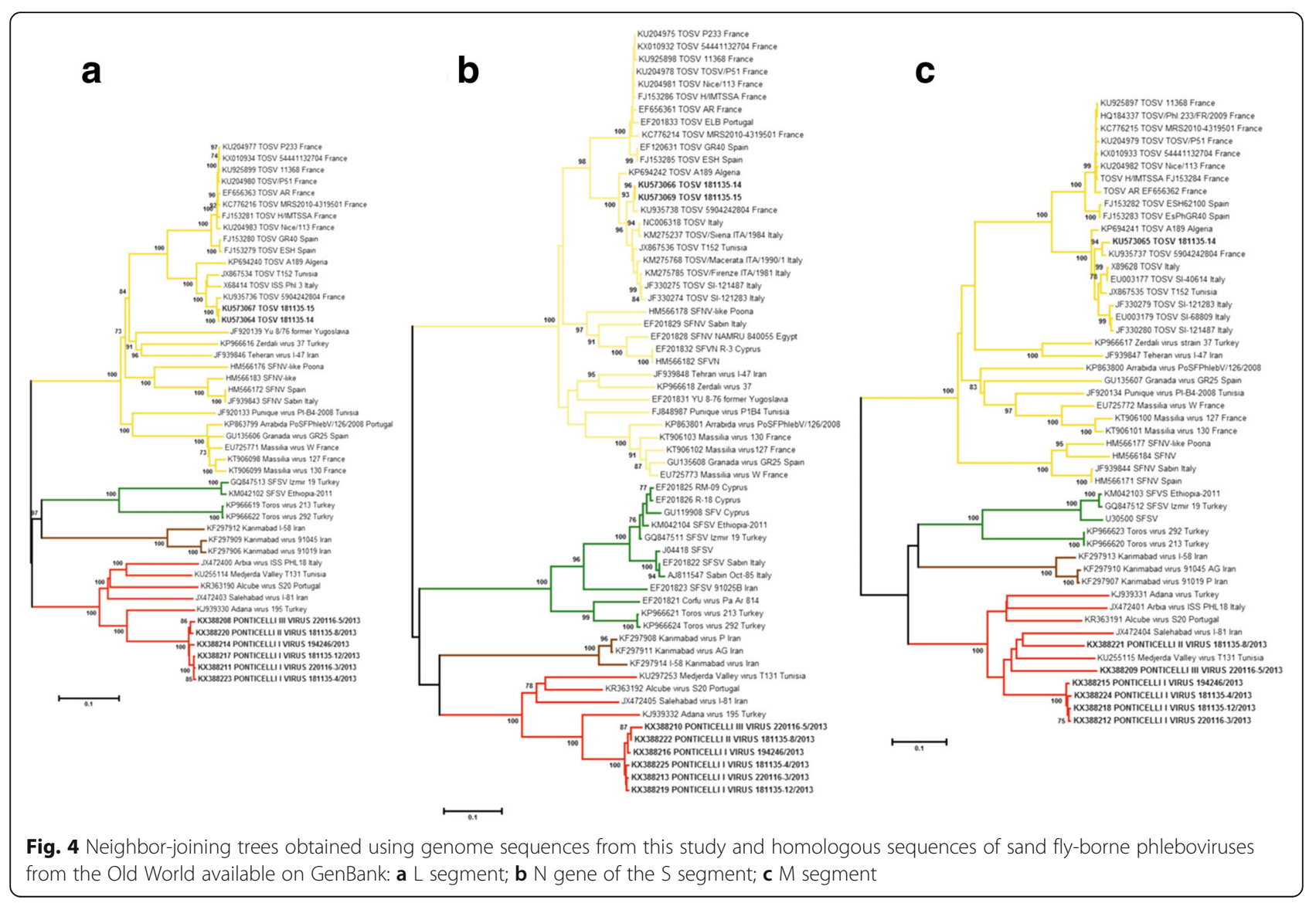


the NS gene (Table 3). These values were lower for the translated amino acid sequences, i.e. $5.1 \%$ for the L segment, $4.6 \%$ for the $\mathrm{N}$ gene, and $4.8 \%$ for the NS gene (Table 3). However, the $3 \mathrm{M}$ segments showed variable differences within the Salehabad phlebovirus species in both the nucleotide (range 23.0-33.7\%) and amino acid (range 12.4-36.4\%) sequences, displaying the smallest differences compared with the Salehabad and the Medjerda Valley viruses (Table 4). The heterogeneity of $M$ segments was evident in similarity analysis of the concatenated genes of the Salehabad phlebovirus species, which showed smallest similarity scores in portion relative to the $\mathrm{Gn}$ and $\mathrm{Gc}$ sequences (Additional file 3: Figure S3).

Apparent recombination events were not detected in the $\mathrm{M}, \mathrm{L}$, and S segments (analyzed as N and NSs sequences) between Ponticelli viruses and homologous sequences of the Salehabad phlebovirus species (data not shown).

\section{Cell culture growth and purification}

The observed CPE on VERO cells was characterized by a diffuse degeneration of the cellular monolayer, with a progressive rounding and subsequent detaching of cells, which appeared numerous and fluctuated in the culture medium. The Ponticelli II and Ponticelli III viruses produced a visible CPE from the third-day post-infection, which appeared complete, with the monolayer almost totally detached on the fifth day. The CPE produced by Ponticelli I was slower; it was visible from the fifth-day post-infection and was complete only after the seventh day.

One of the clones obtained by the purification protocol applied to the Ponticelli III virus (which was recorded in only 1 sample), was subjected to genome sequencing. The 3 segments of the clone had an almost complete identity (over 99.9\%) to the previously obtained sequence, confirming the isolation of a single virus and the reliability of the results obtained.

\section{Discussion}

The fast processing of field-collected sand flies allowed the detection of different phleboviruses: (i) TOSV was detected and isolated in one site; (ii) three closely related phleboviruses of the Salehabad phlebovirus species were detected and isolated in all three sampled sites, and tentatively named Ponticelli I, Ponticelli II and Ponticelli III virus, respectively (according to their different $M$ segments); and (iii) another phlebovirus of the sand fly fever Naples phlebovirus species was detected but not isolated.

The co-circulation of phleboviruses of different species has often been recorded. For example, the Arbia virus and the TOSV were detected in Italy [34], the Saddaguia
Table 3 Pairwise distance matrices of sequences of the $L$ segment, the $\mathrm{N}$ gene and the NS gene of Salehabad phlebovirus species. The upper-right matrix region presents the percentage differences between amino acid alignments; the lower-left, between nucleotide alignments

\begin{tabular}{|c|c|c|c|c|c|c|}
\hline & PONV & ADAV & SALV & ARBV & $\mathrm{ALCV}$ & $\overline{M W}$ \\
\hline \multicolumn{7}{|l|}{ L segment } \\
\hline Ponticelli virus & & 5.1 & 15.7 & 16.6 & 17.4 & 14.5 \\
\hline Adana virus & 19.6 & & 14.5 & 15.1 & 15.5 & 14.1 \\
\hline Salehabad virus & 26.1 & 25.2 & & 10.5 & 11.0 & 9.7 \\
\hline Arbia virus & 26.6 & 26.0 & 23.2 & & 10.7 & 3.8 \\
\hline Alcube virus & 27.1 & 26.5 & 23.9 & 23.3 & & 9.8 \\
\hline Medjerda Valley virus ${ }^{a}$ & 24.9 & 25.3 & 22.6 & 19.1 & 23.3 & \\
\hline \multicolumn{7}{|l|}{ N gene } \\
\hline Ponticelli virus & & 4.6 & 20.3 & 20.3 & 20.3 & 20.3 \\
\hline Adana virus & 16.5 & & 19.6 & 18.3 & 18.3 & 18.3 \\
\hline Salehabad virus & 28.8 & 27.9 & & 11.8 & 9.8 & 11.8 \\
\hline Arbia virus & 27.9 & 28.1 & 24.2 & & 7.2 & 0.0 \\
\hline Alcube virus & 28.4 & 26.6 & 22.5 & 21.9 & & 7.2 \\
\hline Medjerda Valley virus & 27.7 & 28.6 & 23.8 & 6.9 & 21.2 & \\
\hline \multicolumn{7}{|l|}{ NS gene } \\
\hline Ponticelli virus & & 4.8 & 24.9 & 28.2 & 28.2 & 31.1 \\
\hline Adana virus & 17.0 & & 25.3 & 28.9 & 29.0 & 31.9 \\
\hline Salehabad virus & 30.0 & 32.5 & & 30.0 & 30.6 & 28.2 \\
\hline Arbia virus ${ }^{\mathrm{a}}$ & 31.0 & 31.9 & 32.3 & & 27.1 & 2.4 \\
\hline Alcube virus & 31.4 & 32.3 & 33.3 & 33.0 & & 25.6 \\
\hline Medjerda Valley virus & 31.7 & 31.3 & 33.1 & 32.3 & 29.0 & \\
\hline
\end{tabular}

${ }^{\text {a Partial sequences }}$

Note: GenBank accession numbers: Ponticelli virus consensus sequences; Adana virus (ADAV), KJ939330 (L), KJ939332 (N, NS); Salehabad virus (SALV), JX472403 (L), JX472405 (N, NS); Arbia virus (ARBV), JX472400 (L), JX472402 (N, NS); Alcube virus (ALCV), KR363190 (L), KR363192 (N, NS); Medjerda Valley virus (MVV), KU255114 (L), KU297253 (N, NS)

virus and the TOSV in Tunisia [35], the Massilia, the Alcube and the Arrabida viruses in Portugal [9, 21], and the Adana, the Zerdali and the Toros viruses in Turkey [13]. Data obtained confirm that co-circulation of different phleboviruses is a common condition, which implies a very dynamic situation with likely interactions between viruses. The close co-circulation of viruses differing only in the $M$ segments is, however, an uncommon occurrence and highlights the importance of sequencing more than one isolated phlebovirus from any one site whenever possible. The sequence of the $M$ segment of Ponticelli III was confirmed by cloning, and another Ponticelli II virus, with an almost identical $M$ segment, was isolated from sand flies collected in two sites in 2016, one in Valsamoggia municipality (Bologna province), and in Lombardy, a neighbouring region of Emilia-Romagna. These findings demonstrate that three viruses differing in $M$ segments were isolated; these viruses were likely 
Table 4 Pairwise distance matrix of sequences of the M segment of Salehabad phlebovirus species. The upper-right matrix region presents the percentage differences between amino acid alignments; the lower-left, between nucleotide alignments

\begin{tabular}{lllllllll}
\hline & PONVI & PONVII & PONVIII & SALV & MW & ADAV & ARBV & ALCV \\
\hline Ponticelli virus I & & 21.7 & 13.4 & 25.8 & 26.7 & 32.4 & 36.3 & 35.3 \\
Ponticelli virus II $^{\text {a }}$ & 24.1 & & 17.0 & 12.4 & 16.0 & 23.7 & 23.7 & 24.7 \\
Ponticelli virus III & 23.9 & 26.1 & & 26.1 & 28.1 & 31.4 & 36.4 & 35.9 \\
Salehabad virus & 24.6 & 23.0 & 26.5 & & 28.5 & 33.4 & 38.1 & 36.4 \\
Medjerda Valley virus & 26.3 & 23.9 & 25.4 & 24.4 & & 32.0 & 34.8 & 36.0 \\
Adana virus & 27.5 & 30.9 & 29.4 & 29.9 & 31.8 & & 26.1 & 26.9 \\
Arbia virus & 31.1 & 31.8 & 33.7 & 32.8 & 30.4 & 29.4 & & 21.3 \\
Alcube virus & 29.4 & 29.7 & 31.3 & 30.9 & 30.2 & 26.8 & 24.9
\end{tabular}

apartial sequence

Note: GenBank accession numbers: Ponticelli I virus (PONVI), KX388224; Ponticelli II virus (PONVII), KX388221; Ponticelli III virus (PONVIII), KX388209; Salehabad virus (SALV), JX472404; Medjerda Valley virus (MVV), KU255115; Adana virus (ADAV), KJ939331; Arbia virus (ARBV), JX472401; Alcube virus (ALCV), KR363191

the result of reassortment events, as also demonstrated by analysis of the concatenated genes. This mechanism, widely described in the family Bunyaviridae, particularly for the $M$ segment, increases genetic variability through a genetic shift and drives the evolution of these viruses [36]. In sand fly-transmitted phleboviruses, the reassortment of the $M$ segment was already reported in the New World in the Candiru phlebovirus species [7] and was detected in Europe between Massilia, Granada and Arrabida viruses $[8,9]$. The reassortment capacity seems to play a primary role in bunyavirus evolution and in defining the viral pathogenicity of this family [36], as demonstrated by the different abilities of the three Ponticelli viruses to grow on VERO cells, which could indicate a difference in their ability to infect vertebrates. Moreover, the reassortment capacity must be taken into account to avoid misidentification based on serological tests [37].

According to the sequences obtained, the isolated TOSV falls within the lineage A [3, 24], which includes sequences isolated in Tuscany and other Italian regions, confirming the exclusive circulation of viruses from this lineage in Italy. The sequences of the detected, but not isolated, phlebovirus clustered with viruses of the sand fly fever Naples phlebovirus species, which includes viruses with a well-known pathogenic potential [3]. The portion of sequences of this virus alignable with the Fermo virus was small; however, considering the high identity rate, it is likely that the detected sequences could be attributable to the Fermo virus. Nevertheless, longer sequences are necessary to confirm the identification of the detected virus. The failure to isolate this virus was probably due to its low presence in sampled sand flies; in fact, it was detected in only one sample, together with the Ponticelli viruses, which probably displaced this virus from cell culture. Similarly, the Ponticelli viruses were also able to displace the growth of the TOSV in the two TOSV PCR-positive pools collected in August in site 1. Conversely, the
TOSV was isolated in the two pools sampled earlier in the season.

The continuous discovery of new phleboviruses in the Mediterranean Basin indicates the complexity within this genus. This high diversity is probably linked to the intrinsic characteristics of these viruses. In particular, the error-prone nature of the RNA polymerase, induced the high level of nucleotide variation and the relatively low amino acid variation $[3,35]$, as recorded in the obtained sequences. Reassortment or recombination events [9] can also affect this diversity.

The biology of sand flies likely exerts an enhancing effect in generating this wide diversity. Sand flies do not fly far from their breeding sites, and dispersal below one kilometre is usually reported [1]. Thus, the sedentary habit and the focal distribution of these insects favour the geographical isolation of phleboviruses. Even the large population peaks characteristic of sand flies could favour the fast evolution of local viral strains, such as the sympatry of kindred species in a particular focus, like Ph. perfiliewi and Ph. perniciosus, both of the subgenus Larroussius, in this study. The difficulty of finding a vertebrate reservoir in the cycle of sand fly-transmitted phleboviruses may also be an indication of the primary role of sand flies in the persistence of the virus. Some phleboviruses were isolated from rodents in Africa [38], but for most of these viruses, only serological evidence of infection in vertebrates exists. In most cases, infected animals do not show any clinical evidence of infection. It seems that vertebrates act as amplifying hosts but not as reservoirs [38]. In fact, the ability of sand flies to vertically transmit phleboviruses has been widely described and testified by the presence of positive males in different studies; this characteristic further increases the diversifying capacity of sand flies on these viruses. Moreover, multiple infections in arthropods, which do not produce antibodies, could be a favourable occasion for reassortment of the genome segments [36]. All of 
these observations indicate that the evolution and diversity of phleboviruses are strictly linked to the bionomics of sand flies, and to the resulting intensive dynamics of transmission between these viruses and their vectors.

Data on subsamples identified at species level confirmed the overwhelming presence of $\mathrm{Ph}$. perfiliewi among collected sand flies, as already recorded in this area [24], thereby showing that this sand fly is only the probable main vector of the TOSV in the surveyed area, but also the likely main host of the other viruses detected.

Persistence of the TOSV in a particular site was documented by the detection of the TOSV in site 1 , which also showed positive results in the previous year [24]. The virus was detected in this site on two different days of sampling, although with lower infection rates than in 2012, demonstrating the ability of the TOSV to circulate within a particular area persistently.

Despite the TOSV being described as the major cause of summer meningitis in Italy, France and Spain, this virus remains a neglected pathogen. Other pathogens transmitted by sand flies, such as protozoa causing leishmaniasis, are often neglected, though their burdens are increasing worldwide. The growing health importance of these pathogens, which share the same vector, might allow the implementation of a common surveillance strategy.

\section{Conclusions}

The discovery of new phleboviruses reported in this study raises the issue of their infectious potential for humans and animals, since these viruses have often been isolated from vertebrate cell lines (such as Vero cells) and several have been serologically detected in vertebrates. Viruses of the Salehabad phlebovirus species were not linked to disease, but antibodies against the Adana virus were serologically detected with a low prevalence in human and in domestic animal sera (particularly in goats and sheep) in Turkey [20] and human sera in Tunisia [22]. The absence of a specific serological test can impair the detection of phlebovirus infections. Nevertheless, indications of pathogenicity of some of these newly discovered viruses have been reported, such as the detection of a sequence attributable to Adria virus (of the Salehabad phlebovirus species) in a boy with febrile seizure in Greece [39]. Notably, the pathogenic capacity of phleboviruses may be recorded years after their first detection, e.g. the ability of the TOSV to cause disease was discovered approximately fifteen years after the virus was first isolated [40]. The presence of several sand fly-transmitted phleboviruses in a particular area seems to be a common situation in the Mediterranean basin and highlights the need to improve our knowledge of their biology, diagnosis and epidemiology. Therefore, future specific surveillance studies should focus on: (i) isolating the largest number of these viruses for differential diagnoses; (ii) better performing specific serological tests such as the seroneutralization test; (iii) correctly identifying phleboviruses; and (iv) assessing their pathogenic potential. At the same time, and for the same reasons, the biology of phleboviruses should be investigated in depth, and their presence in a particular area should be linked to undiagnosed diseases in humans and other mammals.

\section{Additional files}

Additional file 1: Figure S1. Pictures of two of the sampled sites: site 1 (left) and site 2 (right). (PDF 342 kb)

Additional file 2: Figure S2. Neighbor-joining tree obtained using genome sequences (a, N protein of the S segment; $\mathbf{b}$, complete $\mathrm{M}$ segment). (PDF $153 \mathrm{~kb}$ )

Additional file 3: Figure S3. Similarity plots of the concatenated sequences of the genes of the Ponticelli I, II, and III viruses, and another virus of the Salehabad phlebovirus species. (PDF $427 \mathrm{~kb}$ )

\section{Abbreviations \\ CPE: Cytopathic effect; masl: Meters above sea level; TOSV: Toscana virus}

\section{Acknowledgments}

This paper is dedicated to the memory of our colleague Paolo Cordioli for his fundamental contribution to this research and the study of vector-borne viral infections.

\section{Funding}

This work was partially supported by the Regional project on insect-borne diseases (COMM07001), funded by Regione Emilia-Romagna, and by the grant "Ricerca Corrente" PRC2012002 from the Italian Ministry of Health.

\section{Availability of data and materials}

Sequences obtained during the current study are available in the GenBank database: the 2 Toscana virus strains with accession numbers KU573064, KU573067 (L segments), KU573065, KU573068 (M segments), KU573066, KU573069 (S segments); the Ponticelli I virus strains with accession numbers, KX388211, KX388214, KX388217, KX388223 (L segments), KX388212, KX388215, KX388218, KX388224, (M segments), KX388213, KX388216, KX388219, KX388225 (S segments), the Ponticelli II virus with accession numbers KX388220, KX388221, KX388222, and Ponticelli III virus with accession numbers KX388208, KX388209, KX388210. Nucleotide sequences of the amplicons obtained from field samples by Pan-Phlebo-PCR were stored in GenBank with accession numbers KY354371-KY354394.

\section{Authors' contributions \\ $\mathrm{MC}$ and $\mathrm{PB}$ conceived and designed the experiments. RB and MC sampled sand flies. CC, DL, AL and AM, performed the analyses and experiments. MC, $\mathrm{IB}$ and $\mathrm{PB}$ analyzed the data. MD and SP contributed reagents/materials/ analysis tools. $\mathrm{MC}$ was a major contributor in writing the manuscript. All authors read and approved the final manuscript.}

Ethics approval and consent to participate Not applicable.

Consent for publication Not applicable.

Competing interests

The authors declare that they have no competing interests. 


\section{Publisher's Note}

Springer Nature remains neutral with regard to jurisdictional claims in published maps and institutional affiliations.

\section{Author details}

'Istituto Zooprofilattico Sperimentale della Lombardia e dell'Emilia-Romagna "Bruno Ubertini", Brescia, Italy. ${ }^{2}$ Centro Agricoltura Ambiente "Giorgio Nicoli", Crevalcore, Bologna, Italy.

Received: 2 October 2017 Accepted: 23 January 2018

Published online: 06 February 2018

\section{References}

1. Killick-Kendrick R. The biology and control of phlebotomine sand flies. Clin Dermatol. 1999;17(3):279-89.

2. Maroli M, Feliciangeli MD, Bichaud L, Charrel RN, Gradoni L. Phlebotomine sandflies and the spreading of leishmaniases and other diseases of public health concern. Med Vet Entomol. 2013;27(2):123-47.

3. Alkan C, Bichaud L, de Lamballerie X, Alten B, Gould EA, Charrel RN. Sandfly-borne phleboviruses of Eurasia and Africa: epidemiology, genetic diversity, geographic range, control measures. Antivir Res. 2013;100(1):54-74

4. Bouloy M. Molecular biology of phleboviruses. In: Plyusnin A, Elliott RM, editors. Bunyaviridae molecular and cellular biology. Norfolk: Caister Academic Press; 2011. p. 95-128.

5. Adams MJ, Lefkowitz EJ, King AMQ, Harrach B, Harrison RL, Knowles NJ, et al. Changes to taxonomy and the international code of virus classification and nomenclature ratified by the international committee on taxonomy of viruses (2017). Arch Virol. 2017;162(8):2505-38.

6. Plyusnin A, Beaty BJ, Elliott RM, Goldbach R, Kormelink R, Lundkvist A, et al. Virus taxonomy: Ninth Report of the International Committee on Taxonomy of Viruses. Oxford: Elsevier Academic Press; 2011. p. 725-41.

7. Palacios G, Tesh R, Travassos da Rosa A, Savji N, Sze W, et al. Characterization of the Candiru antigenic complex (Bunyaviridae: Phlebovirus), a highly diverse and reassorting group of viruses affecting humans in tropical America. J Virol. 2011;85(8):3811-20.

8. Collao X, Palacios G, de Ory F, Sanbonmatsu S, Pérez-Ruiz M, Navarro JM, et al. Granada virus: a natural phlebovirus reassortant of the sandfly fever Naples serocomplex with low seroprevalence in humans. Am J Trop Med Hyg. 2010;83:760-5.

9. Amaro F, Hanke D, Zé-Zé L, Alves MJ, Becker SC, Höper D. Genetic characterization of Arrabida virus, a novel phlebovirus isolated in South Portugal. Virus Res. 2016;214:19-25.

10. Papa A, Konstantinou G, Pavlidou V, Antoniadis A. Sandfly fever virus outbreak in Cyprus. Clin Microbiol Infect. 2006;12:192-4.

11. Carhan A, Uyar Y, Ozkaya E, Ertek M, Dobler G, Dilcher M, et al. Characterization of a sandfly fever Sicilian virus isolated during a sandfly fever epidemic in Turkey. J Clin Virol. 2010;48:264-9.

12. Izri A, Temmam S, Moureau G, Hamrioui B, de Lamballerie X, Charrel RN Sandfly fever Sicilian virus, Algeria. Emerg Infect Dis. 2008;14:795-7.

13. Alkan C, Erisoz Kasap O, Alten B, de Lamballerie X, Charrel RN. Sandfly-borne Phlebovirus isolations from Turkey: new insight into the sand fly fever Sicilian and sand fly fever Naples species. PLoS Negl Trop Dis. 2016;10(3): e0004519.

14. Ergunay K, Kasap OE, Orsten S, Oter K, Gunay F, Yoldar AZ, et al. Phlebovirus and Leishmania detection in sandflies from eastern Thrace and northern Cyprus. Parasit Vectors. 2014;7:575.

15. Zhioua E, Moureau G, Chelbi I, Ninove L, Bichaud L, Derbali M, et al. Punique virus, a novel phlebovirus, related to sandfly fever Naples virus, isolated from sandflies collected in Tunisia. J Gen Virol. 2010;91:1275-83.

16. Charrel RN, Moureau G, Temmam S, Izri A, Marty P, Parola P, et al. Massilia virus, a novel Phlebovirus (Bunyaviridae) isolated from sandflies in the Mediterranean. Vector Borne Zoonotic Dis. 2009:9:519-30.

17. Remoli ME, Fortuna C, Marchi A, Bucci P, Argentini C, Bongiorno G, et al. Viral isolates of a novel putative phlebovirus in the Marche region of Italy. Am J Trop Med Hyg. 2014;90(4):760-3.

18. Peyrefitte CN, Grandadam M, Bessaud M, Andry PE, Fouque F, Caro V, et al. Diversity of Phlebotomus perniciosus in Provence, southeastern France: detection of two putative new phlebovirus sequences. Vector Borne Zoonotic Dis. 2013;13(9):630-6.
19. Fares W, Charrel RN, Dachraoui K, Bichaud L, Barhoumi W, Derbali M, et al. Infection of sand flies collected from different biogeographical areas of Tunisia with phleboviruses. Acta Trop. 2015;141(Pt A):1-6.

20. Alkan C, Alwassouf S, Piorkowski G, Bichaud L, Tezcan S, Dincer E, et al. Isolation, genetic characterization, and seroprevalence of Adana virus, a novel phlebovirus belonging to the Salehabad virus complex, in Turkey. J Virol. 2015;89(8):4080-91.

21. Amaro F, Zé-Zé L, Alves MJ, Börstler J, Clos J, Lorenzen S, et al. Co-circulation of a novel phlebovirus and Massilia virus in sandflies, Portugal. Virol J. 2015;12(1):174.

22. Bichaud L, Dachraoui K, Alwassouf S, Alkan C, Mensi M, Piorkowski G, et al. Isolation, full genomic characterisation and neutralisation-based human seroprevalence of Medjerda Valley virus, a novel sandfly-borne phlebovirus belonging to the Salehabad virus complex in northern Tunisia. J Gen Virol. 2015;97(3):602-10.

23. Papa A, Velo E, Bino S. A novel phlebovirus in Albanian sandflies. Clin Microbiol Infect. 2011:17(4):585-7.

24. Calzolari M, Angelini P, Finarelli AC, Cagarelli R, Bellini R, Albieri A, et al. Human and entomological surveillance of Toscana virus in the EmiliaRomagna region, Italy 2010 to 2012. Euro Surveill. 2014;19(48):20978.

25. Romi R, Khoury C, Bigliocchi F, Maroli M. Fact sheet on mites and insects of medical importance [Schede guida su acari e insetti di interesse sanitario]. Rome: Istituto Superiore di Sanità; 1994.

26. Dantas-Torres F, Tarallo VD, Otranto D. Morphological keys for the identification of Italian phlebotomine sand flies (Diptera: Psychodidae: Phlebotominae). Parasit Vectors. 2014:7:479.

27. Pérez-Ruiz M, Collao X, Navarro-Marí JM, Tenorio A. Reverse transcription, real-time PCR assay for detection of Toscana virus. J Clin Virol. 2007;39(4):276-81.

28. Lambert AJ, Lanciotti RS. Consensus amplification and novel multiplex sequencing method for $\mathrm{S}$ segment species identification of 47 viruses of the Orthobunyavirus, Phlebovirus, and Nairovirus genera of the family Bunyaviridae. J Clin Microbiol. 2009:47(8):2398-404.

29. Kampmann ML, Fordyce SL, Avila-Arcos MC, Rasmussen M, Willerslev E, Nielsen LP, et al. A simple method for the parallel deep sequencing of full influenza a genomes. J Virol Methods. 2011;178:243-8.

30. Lavazza A, Pascucci S, Gelmetti D. Rod-shaped virus-like particles in intestinal contents of three avian species. Vet Rec. 1990;126:581.

31. Tamura K, Stecher G, Peterson D, Filipski A, Kumar S. MEGA6: Molecular Evolutionary Genetics Analysis Version 6.0. Mol Biol Evol. 2016;30:2725-9.

32. Martin D, Rybicki E. RDP: detection of recombination amongst aligned sequences. Bioinformatics. 2000;16:562-3.

33. Lole KS, Bollinger RC, Paranjape RS, Gadkari D, Kulkarni SS, Novak NG, et al. Full-length human immunodeficiency virus type 1 genomes from subtype C-infected seroconverters in India, with evidence of intersubtype recombination. J Virol. 1999:73(1):152-60.

34. Verani P, Ciufolini MG, Caciolli S, Renzi A, Nicoletti L, Sabatinelli G, et al. Ecology of viruses isolated from sand flies in Italy and characterized of a new Phlebovirus (Arbia virus). Am J Trop Med Hyg. 1988;38(2):433-9.

35. Dachraoui K, Fares W, Bichaud L, Barhoumi W, Beier JC, Derbali M, et al. Phleboviruses associated with sand flies in arid bio-geographical areas of central Tunisia. Acta Trop. 2016:158:13-9.

36. Briese $\mathrm{T}$, Calisher $\mathrm{CH}$, Higgs $\mathrm{S}$. Viruses of the family Bunyaviridae: are all available isolates reassortants? Virology. 2013:446(1-2):207-16.

37. Liu DY, Tesh RB, Travassos da Rosa AP, Peters CJ, Yang Z, et al. Phylogenetic relationships among members of the genus Phlebovirus (Bunyaviridae) based on partial M segment sequence analyses. J Gen Virol. 2003;84(Pt 2):465-73.

38. Ashford RW. Phlebotomus fevers. In: Encyclopedia of arthropodtransmitted infections. UK: CAB International; 2001. p. 397-401.

39. Anagnostou $V$, Pardalos G, Athanasiou-Metaxa M, Papa A. Novel phlebovirus in febrile child, Greece. Emerg Infect Dis. 2011;17(5):940-1.

40. Calisher $\mathrm{CH}$, Weinberg AN, Muth DJ, Lazuick JS. Toscana virus infection in United States citizen returning from Italy. Lancet. 1987;1(8525):165-6. 